\title{
Análisis de la evaluación y las prescripciones morales sobre el comportamiento de las mujeres lideres en la literatura managerial ${ }^{1}$
}

\author{
Evaluation and moral prescriptions analysis on the behavior of \\ women leaders in managerial literature
}

\author{
Maria Medina-Vicent \\ Universitat Jaume I \\ medinam@uji.es
}

Recibido: 13.04 .2018

Aceptado: 30.08 .2018

\section{RESUMEN}

El papel de las mujeres en el mundo de la gerencia parece haberse convertido en uno de los temas centrales de la agenda política y mediática actual. Una de las herramientas de difusión de los modelos de liderazgo asociados a dicho grupo es la literatura gerencial o managerial, que difunde modas de gestión empresarial entre la clase directiva y el público general. Con el objetivo de identificar si el modelo de liderazgo asociado a las mujeres se aleja del rol de género femenino tradicional, trataremos de identificar los valores que se les asignan a través de un análisis de la literatura managerial. Así pues, en el presente artículo se realizará una propuesta de aplicación metodológica de la Appraisal Theory para el estudio de la evaluación y las prescripciones morales contenidas en la literatura managerial.

\section{PALABRAS CLAVE}

Literatura managerial, teoría de la valoración, valores, prescripciones.

${ }^{1}$ Este estudio se inscribe dentro del Proyecto de Investigación Científica y Desarrollo Tecnológico FFI2016-76753-C2-2-P, financiado por el Ministerio de Economía, Industria y Competitividad del Gobierno de España 


\begin{abstract}
The role of women in management world seems to have become one of the central themes of the current political and media agenda. One of the dissemination tools of the leadership models associated with this group is the managerial literature, which spreads management fashions to the directive class and the general public. In order to identify if the roles assigned to women leaders move away from the traditional femenine gender stereotype, we will try to identify the values that are associated to them through an analysis of managerial literature. Thus, in the present article a proposal of methodological application of the Appraisal Theory will be made for the study of the moral prescriptions contained in the managerial literature.
\end{abstract}

\title{
KEY WORDS
}

Representations, social imaginaries, journalistic discourse, criminalization.

\section{INTRODUCCIÓN}

La presencia de las mujeres en los espacios del poder empresarial es cada vez más evidente (INE 2017) ${ }^{2}$. Dicho progresivo incremento les otorga un protagonismo especial en la reflexión sobre los modelos de gestión empresarial actuales, así como los estilos de liderazgo que se llegan a desarrollar en su seno. Nos referimos tanto a las diversas formas en que se dirige una empresa y se configura la toma de decisiones empresariales; como a los valores que orientan el ejercicio del liderazgo. Así pues, cuando tratamos de acercarnos a los discursos sobre la gerencia y el papel que ocupan las mujeres en su desarrollo, debemos abordar la literatura popular del management, también llamada literatura managerial o gerencial $^{3}$ (Lischinsky 2008), que difunde las modas de gestión entre los cuadros de dirección de las empresas. Se trata de obras de gran carácter divulgativo que traducen las teorías económicas a un lenguaje vulgarizado listo para ser rápi-

2 Tal y como se señala en el informe Mujeres y hombres en España (INE 2017): "En el año 2016, el porcentaje de mujeres en el conjunto de Consejos de Administración de las empresas que forman parte del Ibex-35 era del 19,1\%, porcentaje sin duda insuficiente, casi el doble que el del año 2010, y superior en casi un punto al del año 2014. Dicho porcentaje desciende tres puntos respecto al alcanzado en 2015". Así pues, si bien la presencia de las mujeres en los cargos de dirección sigue siendo escasa, parece haber una progresiva evolución con el paso de los años, que esperemos no llegue a estancarse

${ }^{3}$ Los conceptos "literatura popular del management", "literatura managerial" o "literatura gerencial" se refieren a diferentes maneras de denominar un mismo fenómeno: las obras que abordan y difunden entre la clase directiva mundial, las grandes teorías económicas y las modas de gestión de una forma vulgarizada y simplista. A lo largo de este trabajo trataremos dichos conceptos como sinónimos. 
damente consumido por la clase directiva, pero también por el público general (Huczynski 1993; Alonso y Fernández Rodríguez 2018).

De algún modo, dicha literatura representa lo que los autores Boltanski y Chiapello denominaron "nuevo espíritu del capitalismo" (2002), la lógica neoliberal que condiciona las subjetividades actuales y legitima la constitución de la identidad neoliberal. Por tanto, la literatura popular del management es una herramienta de difusión del poder neoliberal y su carácter prescriptivo acaba por formar parte de los discursos económicos y empresariales presentes en el escenario social (Alonso y Fernández Rodríguez 2013). Esto nos lleva a preguntarnos sobre los riesgos que conlleva para la conformación de una gestión ética ${ }^{4}$ este discurso consumido masivamente por parte de directivos/as y estudiantes de Escuelas de Negocios. Y es que en su acepción general pone de relieve la existencia de un modelo de racionalidad económica neoclásica y un sujeto al servicio del sistema capitalista, ajeno a las consecuencias sociales que tienen las decisiones empresariales (Vázquez Mazzini 2017).

En relación, resulta interesante señalar que encontramos una subcategoría o subgénero dentro de la literatura popular del management dirigida expresamente al público femenino que surge a raíz de la consolidación de la presencia de las mujeres en los cargos de dirección. También en este caso dicha literatura funciona como una herramienta de propaganda ideológica para la conformación de las mujeres como "neosujetos" neoliberales determinados por la lógica de la oferta y la demanda. Como señala la autora Elisabeth Kelan (2009: 143) en referencia a las mujeres en el mundo del trabajo, al entrar a formar parte de las filas de la dirección empresarial, las mujeres profesionales también participan de la lógica neoliberal. Y es que, muchas se construyen a sí mismas en términos de mercado y aceptan la inseguridad como parte de su trabajo. Es decir, de algún modo este tipo de literatura nos da las bases para comprender sobre qué premisas se construye a las mujeres como individuos que planifican su vida profesional, pero no solamente ésta, en base a los criterios del mercado (Mavin, Grandy, y Williams 2014; Scharff 2015).

Así pues, en la medida en que dichas obras están dirigidas a las directivas, al analizar sus contenidos podremos acercarnos al conocimiento de los valores asociados al modelo de mujer líder en la empresa. Partiendo del reconocimiento de esta realidad, el objetivo principal de nuestro análisis estriba en identificar el modelo de mujer líder en la empresa que se construye a través de la literatura popular del management dirigida a mujeres profesionales. Cuando hablamos de modelo nos referimos esencialmente a los valores que se desprenden de los comportamientos y acciones que se les aconseja llevar a cabo desde este tipo de obras. De este modo, el trabajo de análisis que llevaremos a cabo nos debe permitir identificar los presupuestos sobre los que se aconseja a las mujeres que

${ }^{4}$ Por "gestión ética de la empresa" entendemos un modelo de gestión que tenga en cuenta los intereses de todos los grupos afectados por la actividad de la empresa. Es decir, una gestión que reconozca el valor social de las empresas y que promueva el ejercicio moral en su toma de decisiones (Cortina 1994; Conill 2003; García-Marzá 2004). 
desarrollen sus liderazgos en la alta dirección empresarial. Concretamente, pretendemos identificar: los valores asociados al modelo de mujer líder; tanto los asociados por naturaleza/biología, como los valores que se pueden ejercitar en el desarrollo del liderazgo. Como se puede observar, dentro de los valores diferenciamos dos tipos con la intención de identificar si el carácter de construcción de dicho modelo de liderazgo es más bien de corte esencialista, o de corte constructivista; ya que esto también nos dirá mucho sobre el estado de la igualdad de género en la empresa y las posibilidades de su transformación.

Resultará especialmente interesante identificar el lenguaje prescriptivoimperativo y evaluativo que puedan contener dichas obras, ya que serán estos fragmentos lingüísticos los que contengan los marcadores enunciativos referidos a comportamientos, acciones y valores sobre los que se edifica la noción de mujer líder empresarial. Para identificar dichos componentes, nos centraremos en la Appraissal Theory (Christie y Martin 1997; Eggins y Slade 1997; Martin 2000; Martin y Rose 2003; Martin y White 2005; White 2002), que ofrece un modelo estándar para el análisis de la evaluación en el discurso. Consideramos que dicha metodología nos debe permitir identificar los presupuestos sobre los que se aconseja a las mujeres que desarrollen sus liderazgos en el ámbito de la alta dirección empresarial, es decir, el análisis de la evaluación y las prescripciones morales sobre el comportamiento de las mujeres líderes en la literatura managerial. Por esta razón, en el presente escrito realizaremos nuestra propia propuesta de aplicación de dicha metodología al estudio de la evaluación y las prescripciones morales en la literatura managerial.

\section{DISCURSOS E IDEOLOGÍA}

En nuestra investigación optamos por realizar un análisis del discurso de una muestra de la literatura popular del management dirigida a mujeres, que se centre en la identificación y estudio de aquellos componentes que nos permitan identificar los valores sobre los que se construye el modelo de mujer líder dentro de dicha literatura. Así, más allá de poner el foco de atención en el aspecto estructural, nos centraremos en el discernimiento de los discursos y la ideología impresos en las obras. Debemos tener en cuenta que el discurso dentro de la organización está monopolizado por la dirección y este privilegio es considerado por el resto de grupos como algo natural y neutral en lo que respecta a valores (Alvesson y Willmott 1992: 24). Sin embargo, en la medida en que la dirección ha sido tradicionalmente asociada a lo masculino, dichos valores masculinizados y en muchas ocasiones excluyentes (Benschop y Doorewaard 1998; Due Billing 2011; Kenny y Fotaki 2015), habrán calado en el imaginario de la clase directiva perpetuando una cultura patriarcal. Por esta razón, nos interesa desvelar los valores que hay tras la pretendida neutralidad de dicho discurso, y cómo influyen en un tema tan importante como la desigualdad de género en la empresa.

Como se puede observar en la figura, los textos se inscriben dentro de prácticas discursivas, que a su vez se encuentran en el seno de las prácticas sociales 
(Fairclough 1992a, 1992b, 2003). De este modo, las obras del management están dentro del discurso empresarial, que a su vez está emplazado en el discurso sobre la organización del trabajo (Fernández Rodríguez 2007a, 2007b). Dichas obras son la codificación en textos del discurso sobre la organización del trabajo que tiene la empresa y que vertebra a su vez el espacio social. Si incorporamos la perspectiva de género a dicha cuestión, veremos que la literatura popular del management dirigida a mujeres se sitúa dentro del discurso empresarial sobre las mujeres líderes, y a su vez, dentro del discurso empresarial sobre la igualdad de género.

Y es que, la perspectiva de género se alza como una mirada crítica al mundo (Medina-Vicent y Reverter-Bañón 2016), una mirada que aplicada a dicho análisis nos permite identificar los núcleos discursivos de perpetuación de la desigualdad de género y de poder dentro de las organizaciones empresariales. Componentes todos que no podemos desligar de su marco de sentido general referido a la división sexual del trabajo sobre la que se sustentan nuestras sociedades. De este modo, con el análisis del discurso se obtendrá información no solamente de las obras concretas, sino del marco de sentido, es decir de los discursos, en el que se produce y acoge dicha producción textual.

Figura 1. Literatura, discurso, estructura (elaboración propia)

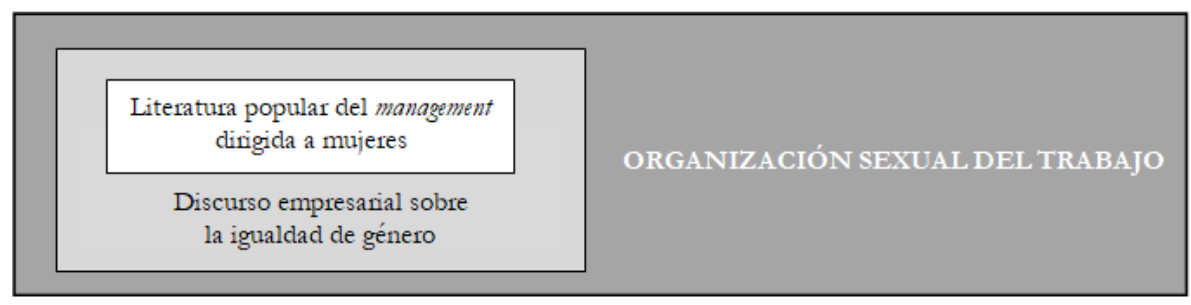

A la hora de analizar los textos debemos tener en cuenta que, en el proceso de configuración enunciativa, el hablante selecciona el discurso y el tipo de texto, elige su contenido y aplica diferentes mecanismos de textualidad que dan forma, identidad e individualidad al texto concreto. Por tanto, en el texto se encuentran inscritas marcas enunciativas que nos pueden aportar información sobre los discursos referidos al modelo de mujer líder que se construye en este tipo de obras (Alonso 1998). En este análisis rastrearemos las huellas enunciativas que existen en el texto y que nos hablan acerca de las prescripciones comportamentales que se lanzan hacia las mujeres profesionales, que a su vez nos aportarán información sobre el estado de la igualdad de género en la empresa.

Cabe señalar que en estudios previos sobre literatura gerencial se han empleado otras metodologías de análisis que también pueden ser oportunas para analizar otros aspectos de dicha literatura que van más allá de los valores morales y las prescripciones, aunque también las tengan en cuenta. Encontramos trabajos como los de Norman Fairclough (1992a, 1992b, 2003) y Éve Chiapello 
(2002); o el trabajo de éste último junto a Luc Boltanski (2002). En el escenario nacional merecen especial mención los estudios sociológicos de Carlos Jesús Fernández Rodríguez (2007a) junto a Luis Enrique Alonso (2013, 2018)5 .

\section{APROXIMACIÓN A LOS PRINCIPIOS DE LA APPRAISAL THEORY}

La Appraisal Theory o Teoría de la Valoración (Christie y Martin 1997; Eggins y Slade 1997; Martin 2000; Martin y Rose 2003; Martin y White 2005; White 2002), ofrece un modelo estándar para el análisis de la evaluación en el discurso. Esta teoría surge en el marco de la Lingüística Sistémica-Funcional durante las décadas de los ochenta y noventa del siglo veinte (Iedema, Feez, y White 1994), y su principal empuje fue el proyecto Write It Right del Programa NSW Disadvantaged Schools.

Según James R. Martin, la Appraisal Theory se refiere a los recursos semánticos utilizados para negociar emociones, juicios y valoraciones, así como recursos para amplificar y comprometerse con estas evaluaciones (2000: 145). Por tanto, dicha teoría explora en qué contextos, de qué manera y qué recursos lingüísticos expresan, negocian y naturalizan posiciones intersubjetivas e ideológicas de los hablantes. Se ocupa de los significados que modifican los términos del compromiso del hablante con sus emisiones, es decir, que modifican lo que está en juego en la relación interpersonal, tanto en las emisiones individuales como en el desarrollo del texto. Dicha metodología nos remite directamente a las negociaciones sobre juicios y valoraciones de los hablantes y los textos. Por tanto, la teoría de la valoración es un método eficaz para analizar cómo los valores morales se inscriben y codifican a través de los textos (Morrish y Sauntson 2013). Cabe señalar que el modelo concreto en el que nos centraremos será el desarrollado por Martin y White (2005), que diferencia tres subsistemas: attitude, engagement y graduation:

Attitude is concerned with our feelings, including emotional reactions, judgements of behaviour and evaluation of things. Engagement deals with sourcing attitudes and the play of voices around opinions in discourse. Gra-

${ }^{5}$ Se ha escogido la Appraisal Theory como metodología de análisis frente a otras posibilidades como el Análisis Crítico del Discurso (Fairclough 1992a, 1992b, 2003), las cités utilizadas por Boltanski y Chiapello (2002), o aproximaciones más sociohermenéuticas (Alonso y Fernández Rodríguez 2006, 2013, 2018), debido a la naturaleza interdisciplinar del trabajo realizado, que transita entre el ámbito de la sociología, la ética empresarial y la filosofía feminista. Así pues, los principios de la Appraisal Theory se muestran más coherentes con los objetivos definidos en esta investigación. Si bien otras metodologías como las citadas podrían haber arrojado conclusiones interesantes sobre el fenómeno estudiado, nuestro objetivo de abordar la dimensión prescriptiva de los textos -y normativa desde un punto de vista ético- se alineaba mejor con la capacidad de la Appraisal Theory para identificar no solamente las prescripciones dentro de los textos, sino también el componente valorativo y normativo de los mismos, enmarcándonos en un contexto social de corrección y normatividad del comportamiento concretos. 
duation attends to grading phenomena whereby feelings are amplified and categories blurred. Attitude is itself divided into three regions of feeling, affect, judge-ment and appreciation. Affect deals with resources for construing emotional reactions (Martin y White 2005: 34-35).

En primer lugar, la actitud (Martin y White 2005: 42-91) se refiere a los significados mediante los cuales los textos y los/as lectores/as otorgan un valor intersubjetivo a sus juicios y las respuestas emocionales/afectivas que asocian con los participantes y los procesos en los que se encuentran involucrados, ya sea relacionados con respuestas emocionales o con sistemas de valores culturalmente determinados. A su vez, la actitud se divide en tres subsistemas: affect o afecto (caracterización de los fenómenos en relación con la emoción), judgement o juicio (la evaluación del comportamiento humano en relación con las normas sociales) y appreciation o apreciación (evaluación de objetos y productos en relación con principios estéticos y otros sistemas de valor social). Dentro de la categoría de la actitud, el juicio nos interesa especialmente como subcategoría, por esta razón, lo trataremos más adelante con mayor profundidad.

En segundo lugar, encontramos el engagement o compromiso (Martin y White 2005: 92-161) referido a los recursos utilizados por el hablante para posicionar su voz en relación con las proposiciones de un texto, es decir, los significados por medio de los cuales los hablantes reconocen o ignoran la diversidad de puntos de vista que sus emisiones ponen en juego y por medio de las cuales negocian un espacio interpersonal para sus propias posiciones dentro de esa diversidad. Dichos recursos pueden ser: modalizadores de probabilidad (perhaps, it may..., I think..., surely), de fase o etapa de realidad (it seems), de atribución (his alleged..., informed sources report..., scientists have found evidence suggesting that); de proclamación (In fact, I am compelled to conclude..., It is true...); de expectativa (predictably, of course) y de contra-expectativa (amazingly) (White 2000).

En tercer lugar, encontramos la graduation o gradación (Martin y White 2005: 135-141), que se refiere a los valores por medio de los cuales los hablantes gradúan el impacto interpersonal de sus emisiones, aumentan o disminuyen la fuerza de sus emisiones; al tiempo que gradúan (desdibujan o agudizan) el foco de sus categorizaciones semánticas. En cuanto a la fuerza de la enunciación, podemos encontrarla reflejada en el uso de adverbios como slightly, somewhat, very, completely; mientras que el foco lo podemos ver reflejado en el siguiente ejemplo: I was feeling kind'v woozy, they effectively signed his death warrant; a true friend, pure folly (Martin y White 2005: 137-138).

A la hora de aplicar el marco metodológico que nos presta dicha teoría en nuestro análisis, debemos tener en cuenta que lo que se considera evaluación dentro de un texto depende directamente del campo en el que se inscribe dicho discurso en cuestión. Por esta razón, la Appraisal Theory requiere que el/ la analista sea especialmente sensible al contexto particular en que el texto se inscribe. Y es que, a la hora de identificar los marcadores de valoración en un texto el analista debe contar con un profundo conocimiento de las prácticas institucionales implicadas en los textos que se están estudiando y tener en cuenta las 
actitudes que se espera de los/as lectores/as respecto a dichas prácticas (Morrish y Sauntson 2013: 67). Porque algunos de los marcadores de la actitud, por ejemplo, dependen de donde se sitúan en el contexto que otorga sentido al texto. Por tanto, como analistas, necesitaremos estar pendientes de aquellos elementos que nos remiten directamente al contexto de acción.

En base a nuestros conocimientos sobre la literatura del management dirigida a mujeres (Medina-Vicent 2018a) y debido a la gran amplitud del marco metodológico que nos presta la Appraisal Theory, nos centraremos solamente en aquellos aspectos que están directamente relacionados o que resultan más relevantes para los objetivos de nuestra investigación. Es decir, las subcategorías del juicio y la apreciación que se encuentran dentro de la categoría de la actitud, ya que es a partir de estas cuestiones donde podremos encontrar los elementos que nos interesa analizar: lenguaje prescriptivo-imperativo, lenguaje evaluativo $\mathrm{y}$ aseveraciones tajantes sobre las diferencias entre mujeres y hombres.

Así pues, cabe retomar aquí la categoría de la actitud que explicábamos en párrafos anteriores, y dentro de la cuál Martin (2005) identifica tres subsistemas de la posición actitudinal del hablante: afecto, que se refiere a los recursos lingüísticos utilizados para construir las respuestas emocionales; el juicio, los recursos lingüísticos empleados para construir las evaluaciones morales o sociales sobre el comportamiento, y la apreciación, los recursos lingüísticos usados para construir las cualidades estéticas de los procesos. A continuación podemos consultar algunos ejemplos tomados de Morrish y Sauntson (2013: 65) para ilustrar los recursos a los que nos estamos refiriendo:

AFECTO - [Our students] will be inspired to acquire the knowledge, skills and confidence.

JUICIO - [A university] dedicated to academic achievement.

APRECIACIÓN - one of the worlds's leading research and teaching universities.

Nos interesa el subsistema actitudinal del juicio, ya que comprende significados que sirven para evaluar positiva o negativamente el comportamiento humano, en relación con un conjunto de normas institucionalizadas. Así, el juicio está involucrado cuando el hablante evalúa a un participante en relación con sus actos o disposiciones (Iedema, Feez, y White 1994). Al mismo tiempo, las normas sociales que se ponen en juego en estas evaluaciones de juicio pueden contener tanto la forma de reglas como de expectativas sociales y sistemas de valores menos definidos. De este modo, con el juicio podemos evaluar el comportamiento como moral o inmoral, legal o ilegal, aceptable o inaceptable, etc. Estas son cuestiones que nos resultan especialmente reveladoras por lo que se refiere a la identificación del lenguaje prescriptivo-imperativo y evaluativo.

Y es que el juicio se refiere a cómo comportarse (Martin y White 2005: 53), y contiene los recursos lingüísticos mediante los cuales se evalúa un comportamiento en base a principios normativos válidos en un sistema social concreto. En este sentido, el hablante efectúa una valoración del comportamiento y provee normas, prescribe utilizando el lenguaje para posicionarse en un contexto social. 
En el caso que nos ocupa, poder identificar el carácter prescriptivo de la literatura popular del management hacia el comportamiento de las mujeres líderes resulta clave, ya que de este modo podremos estar más cerca de conocer cómo el mundo de la gestión pide a las mujeres comportarse de un cierto modo. Al igual que todas las categorías de la Appraisal Theory, el juicio funciona en una escala de positivo a negativo, y sus dimensiones se dividen en dos áreas: social esteem (estima social) y social sanction (sanción social) (Martin 2000: 53-56; Martin y White 2005).

La estima social se refiere a las marcas sociales que identifican las evaluaciones sobre cómo el comportamiento alcanza o no el estándar social esperado en una cultura determinada. Este tipo de marcadores evalúan instituciones, sujetos individuales y comportamientos en términos de su normalidad (cómo de usuales o inusuales son en un contexto determinado); capacidad (cómo de capaces son) y tenacidad (cómo de determinados o resolutivos son) (Martin y White 2005: 53). La sanción social se refiere a instituciones individuales y comportamientos en términos de veracidad (cómo de veraces son) y propiedad (cómo de éticos son). Además, provee de marcas evaluativas que indican si un comportamiento es visto como correcto o incorrecto. Como se puede deducir de lo que acabamos de explicar, nos interesarán los marcadores referidos a la sanción social, ya que evalúan la corrección de los comportamientos.

Irremediablemente, el subsistema del juicio nos lleva a hablar del de la valoración o apreciación (Martin y White 2005: 56), otro de los subsistemas de la actitud que expresa evaluaciones positivas y negativas sobre textos, procesos y fenómenos. Cabe señalar que se encuentra organizada en tres variables: reaction (reacción), que es el grado en que dicho texto/proceso/fenómeno captura el impacto emocional que tiene en nosotros/as; composition (composición), nuestras percepciones de proporcionalidad (balance) y detalle (complejidad del texto y/o proceso); y, por último, la valuation (valoración) que es la evaluación del significado social del texto-proceso-fenómeno.

Según Martin, tanto el juicio como la apreciación codifican e institucionalizan sentimientos. Así pues, y tal y como se puede leer en el gráfico de la siguiente página, el afecto, el sistema básico de la actitud, es luego institucionalizado en juicio y apreciación. Y es que, el juicio institucionaliza sentimientos como proposiciones sobre el comportamiento, mientras que la apreciación institucionaliza los sentimientos como proposiciones sobre las cosas (Martin 2000: 147). Así, la apreciación es una reacción porque aquello que está siendo evaluado es un fenómeno del entorno. Por ejemplo, si describimos algo como "excitante", implica que nos sentimos excitados, por tanto, la evaluación contiene un elemento de afecto, que es institucionalizado en una evaluación sobre algo como "excitante". Consecuentemente, a través del juicio se establecen significaciones sobre lo bueno y lo malo: "judgement deals with attitudes towards behaviour, which we admire or criticise, praise or condemn" (Martin y White 2005: 42).

Si tenemos en cuenta que el juicio nos remite a los significados compartidos por una comunidad, entenderemos que en el caso de la literatura popular del management dirigida a mujeres profesionales, dicho lenguaje nos remitirá 
a los significados compartidos por la organización sobre aspectos relacionados con la cuestión de género y/o la presencia de las mujeres en el ámbito empresarial. Aportará información sobre las conductas que una mujer profesional debe incorporar para integrarse en la lógica de la organización. Por esta razón, Painter (2003) señala que los juicios referidos a las normas éticas, acaban por institucionalizarse y afectan la apreciación del sujeto sobre las proposiciones, condicionando que éste efectúe una valoración de las mismas como buenas o malas. Y es que, al final, las propias directrices que se les comunican a dichas mujeres a través de este tipo de obras, acabarán afectando a su valoración sobre su presencia en la empresa, así como a su percepción del estado de la igualdad de género en la misma, pudiendo llegar a transformar su visión del fenómeno y su discurso sobre el mismo.

\section{LA APLICACIÓN DE LA APPRAISAL THEORY AL ANÁLISIS DE LA LITERATURA GERENCIAL}

Una vez tratada la base metodológica de la Appraisal Theory, identificaremos los indicadores utilizados en la realización de nuestro análisis de las obras de la literatura popular del management dirigida a mujeres ${ }^{6}$, basándonos principalmente en las categorías de análisis del juicio (sanción social) y la apreciación. Las categorías que proponen Martin y White (2005) para la identificación de dichas dimensiones se disponen en la tabla número uno, que se puede consultar a continuación. Teniendo en cuenta estos términos, y en base al objetivo de la presente investigación elaboraremos nuestra propia propuesta de indicadores.

\begin{tabular}{|l|l|l|}
\hline \multicolumn{3}{|c|}{ Tabla 1. Juicio - Sanción social y Apreciación } \\
\hline $\begin{array}{l}\text { JUDGEMENT } \\
\text { - SOCIAL SANC- } \\
\text { TION }\end{array}$ & Positive (praise) & Negative (condemn) \\
\hline $\begin{array}{l}\text { Veracity (truth) } \\
\text { Howhonest? }\end{array}$ & $\begin{array}{l}\text { truthful, honest, cred- } \\
\text { ible...; } \\
\text { frank, candid, direct...; } \\
\text { discrete, tactful... }\end{array}$ & $\begin{array}{l}\text { dishonest, deceitful, lying...; } \\
\text { deceptive, manipulative, devi- } \\
\text { ous...; } \\
\text { blunt, blabbermouth... }\end{array}$ \\
\hline
\end{tabular}

${ }^{6}$ Se han decidido mantener tanto los indicadores del análisis como el material discursivo en inglés, ya que el análisis se ha efectuado sobre las obras en su original en inglés. 


\begin{tabular}{|c|c|c|}
\hline $\begin{array}{l}\text { Propriety (ethics) } \\
\text { How far beyond re- } \\
\text { proach? }\end{array}$ & $\begin{array}{l}\text { good, moral, ethical...; } \\
\text { law abiding, fair, just...; } \\
\text { sensitive, kind, caring...; } \\
\text { unassuming, modest, } \\
\text { humble...; } \\
\text { polite, respectful, rever- } \\
\text { ent...; } \\
\text { altruistic, generous, chari- } \\
\text { table... }\end{array}$ & $\begin{array}{l}\text { bad, immoral, evil...; } \\
\text { corrupt, unfair, unjust...; } \\
\text { insensitive, mean, cruel...; } \\
\text { vain, snobby, arrogant...; } \\
\text { rude, discourteous, irreverent...; } \\
\text { selfish, greedy, avaricious... }\end{array}$ \\
\hline $\begin{array}{l}\text { Modulations of } \\
\text { obligation (Modu- } \\
\text { lations of obligation } \\
\text { can be related to } \\
\text { lexicalised judge- } \\
\text { ments of propriety) }\end{array}$ & \multicolumn{2}{|c|}{$\begin{array}{l}\text { Go. } \\
\text { You should go. } \\
\text { You're supposed to go. } \\
\text { It's expected you'll go. } \\
\text { It'd be unfair for you to go. } \\
\text { It'd be corrupt, insensitive, arrogant, selfish, rude, etc. [judge- } \\
\text { ment: propriety] } \\
\text { Reasoning }\end{array}$} \\
\hline APPRECIATION & Positive & Negative \\
\hline $\begin{array}{l}\text { Reaction: } \\
\text { impact 'did it grab } \\
\text { me? }\end{array}$ & $\begin{array}{l}\text { arresting, captivating, en- } \\
\text { gaging...; } \\
\text { fascinating, exciting, mov- } \\
\text { ing...; } \\
\text { lively, dramatic, in- } \\
\text { tense...; } \\
\text { remarkable, notable, sen- } \\
\text { sational...; }\end{array}$ & $\begin{array}{l}\text { dull, boring, tedious...; } \\
\text { dry, ascetic, uninviting...; } \\
\text { flat, predictable, monotonous...; } \\
\text { unremarkable, pedestrian... }\end{array}$ \\
\hline $\begin{array}{l}\text { Reaction: } \\
\text { quality 'did I like } \\
\text { it?' }\end{array}$ & $\begin{array}{l}\text { okay, fine, good... } \\
\text { lovely, beautiful, splen- } \\
\text { did...; } \\
\text { appealing, enchanting, } \\
\text { welcome... }\end{array}$ & $\begin{array}{l}\text { bad, yuk, nasty...; } \\
\text { plain, ugly, grotesque...; } \\
\text { repulsive, revolting, off-put- } \\
\text { ting... }\end{array}$ \\
\hline $\begin{array}{l}\text { Composition: } \\
\text { balance 'did it hang } \\
\text { together? }\end{array}$ & $\begin{array}{l}\text { balanced, harmonious, } \\
\text { unified, symmetrical, pro- } \\
\text { portioned...; } \\
\text { consistent, considered, } \\
\text { logical...; } \\
\text { shapely, curvaceous, wil- } \\
\text { lowly... }\end{array}$ & $\begin{array}{l}\text { unbalanced, discordant, irregu- } \\
\text { lar, } \\
\text { uneven, flawed...; } \\
\text { contradictory, disorganised...; } \\
\text { shapeless, amorphous, dis- } \\
\text { torted... }\end{array}$ \\
\hline $\begin{array}{l}\text { Composition: Com- } \\
\text { plexity } \\
\text { "was it hard to fol- } \\
\text { low? }\end{array}$ & $\begin{array}{l}\text { simple, pure, elegant...; } \\
\text { lucid, clear, precise...; } \\
\text { intricate, rich, detailed, } \\
\text { precise... }\end{array}$ & $\begin{array}{l}\text { ornate, extravagant, byzan- } \\
\text { tine...; } \\
\text { arcane, unclear, woolly...; } \\
\text { plain, monolithic, simplistic... }\end{array}$ \\
\hline
\end{tabular}




\begin{tabular}{|c|c|c|}
\hline $\begin{array}{l}\text { Valuation: 'was it } \\
\text { worthwhile?' }\end{array}$ & $\begin{array}{l}\text { penetrating, profound, } \\
\text { deep...; } \\
\text { innovative, original, cre- } \\
\text { ative...; } \\
\text { timely, long awaited, land- } \\
\text { mark...; } \\
\text { inimitable, exceptional, } \\
\text { unique...; } \\
\text { authentic, real, genuine...; } \\
\text { valuable, priceless, worth- } \\
\text { while...; } \\
\text { appropriate, helpful, effec- } \\
\text { tive... }\end{array}$ & $\begin{array}{l}\text { shallow, reductive, insignifi- } \\
\text { cant...; } \\
\text { derivative, conventional, pro- } \\
\text { saic...; } \\
\text { dated, overdue, untimely...; } \\
\text { dime-a-dozen, everyday, com- } \\
\text { mon; } \\
\text { fake, bogus, glitzy...; } \\
\text { worthless, shoddy, pricey...; } \\
\text { ineffective, useless, write-off... }\end{array}$ \\
\hline
\end{tabular}

Fuente: The Language of Evaluation: The Appraisal Framework (Martin y White 2005: 53-57)

En primer lugar, identificaremos los mandatos, enunciados imperativos y/o prescripciones que se establecen en dichas obras, que pueden referirse a comportamientos, acciones y/o decisiones que se aconseja desarrollar en su práctica profesional a las mujeres profesionales. Es en este tipo de lenguaje donde encontramos los consejos sobre comportamientos que deben desarrollar las mujeres profesionales en su liderazgo, y de ahí, los valores sobre los que dichos modelos de comportamiento se construyen. Así pues, el lenguaje imperativo-prescriptivo está orientado a la acción y contiene una intención normativa, es decir, pretende establecer una serie de normas de actuación para el/la lector/a.

Por esta razón, el enunciado imperativo o prescriptivo no tiene función primaria informativa; es decir, no es ni verdadero ni falso, pues no se refiere a un hecho dado, sino que busca guiar el comportamiento del receptor en una situación determinada. En resumen, en este tipo de lenguaje podemos encontrar los valores que pretendemos identificar, ya que a través de él se tiende a cambiar la situación existente, motiva o provoca la realización de una conducta en un cierto sentido. Esto es lo que ocurre con las obras de la literatura popular del management dirigidas a mujeres, ya que buscan transformar la situación de la mujer en la empresa, aportándole consejos y guías.

Además, cabe señalar que en los textos prescriptivos predominan tanto la función apelativa como la representativa, ya que se utiliza el lenguaje para influir en la conducta del receptor y se aporta información para llevar a cabo la acción prescrita. Respecto a las características lingüísticas de los fragmentos prescriptivos, cabe señalar que suelen estar escritos en un lenguaje claro, preciso y sencillo que puede ser comprendido fácilmente. Por esta razón, resulta frecuente encontrar tanto explicaciones breves e imágenes que aclaren el contenido del texto, como marcadores que expresan orden. Al mismo tiempo, las formas verbales más frecuentes son las imperativas y los infinitivos. 
En este sentido, las prescripciones pueden ser imperativas, es decir, pueden adoptar la forma de una orden, pero también puede formularse con un nivel mayor de permisibilidad. Así pues, en nuestra identificación del lenguaje prescriptivo, la Appraisal Theory nos presta las directives and the modality of permission/ obligation (Martin y White 2005: 110), es decir, las directrices de modalidad de permiso y obligación. En esta categoría se incluyen las locuciones referidas al permiso y la obligación, conocida como la modalidad deóntica. Los modales deónticos abren el diálogo a diferentes posibilidades, mientras que el lenguaje imperativo es monoglósico y no da lugar a alternativas de acción (Martin y White 2005: 111). A nosotros nos interesa tanto un tipo de lenguaje como el otro, en la medida en que el imperativo ofrece órdenes de comportamiento, mientras que los modales deónticos ofrecen una posibilidad de elección al/la lector/a, estableciendo una recomendación de comportamiento, por lo que se mueve en un marco moral también referido a la acción.

Teniendo en cuenta estas cuestiones, se prestará especial atención a los verbos modales have to, must,ought to, should, can, could y do - también en sus formas negativas - utilizados para expresar necesidad u obligatoriedad, $\mathrm{y}$ recomendaciones. Identificar los enunciados que contienen dichas fórmulas nos permitirá deducir las acciones y normas que se aconsejan a las mujeres profesionales a través de este tipo de obras. Al mismo tiempo, este tipo de enunciados nos permitirán ver cuáles son los valores contenidos en dichas afirmaciones prescriptivas. También resultará importante saber identificar los enunciados exclamativos, que instan a la acción del/la lector/a.

En segundo lugar, deberemos identificar los enunciados que nos remiten al lenguaje evaluativo, es decir, aquellos fragmentos del texto y enunciados que nos indiquen qué acciones y decisiones son buenas, y cuáles son malas para el futuro de las mujeres profesionales en la empresa. Se trata de aquellos fragmentos más relacionados con lo que debe o no debe hacer una mujer líder en el desarrollo de su cargo de dirección. Este tipo de lenguaje que nos remite a una dimensión moral de los comportamientos, se encuentra más relacionada con el "debería ser" que con un discurso totalmente imperativo. Recordamos que para identificar este tipo de lenguaje, y siguiendo con la Appraisal Theory, tendremos en cuenta las categorías de juicio (sanción social) y apreciación (Martin y White 2005: 53). Así, nos centraremos en los términos que nos remitan a lo bueno y lo malo.

En cuanto a los términos positivos, trataremos de identificar: good, ok, right, value, nice, convinient, adequate, satisfying, possible... y los negativos bad, wrong, mistake, error, failure, dissatisfying, imposible... Dejamos abierta esta cuestión para incorporar enunciados que nos puedan remitir a aquello considerado bueno o malo, pero que no utilice estos conceptos. De este modo, identificando aquellos comportamientos que se consideran correctos e incorrectos para las mujeres que ocupan dichas posiciones de responsabilidad empresarial, estaremos más cerca de saber qué valores se consideran positivos en dicho modelo de mujer líder, y qué tipo de valores no.

Al mismo tiempo, debemos tener en cuenta que si uno de nuestros objetivos es diferenciar entre los valores que se asocian a las mujeres líderes de forma 
esencialista, y aquellos que se les insta a aprender porque no conforman parte de dicha "esencia". Deberemos prestar especial atención al uso de los vocablos natural, naturally, tend to, tendency, general, generally, inclination, incline, common, commonlyusually, hardwired, born... Es decir, aquellas palabras que nos remitan a una naturaleza irrenunciable de las mujeres. Nos interesan las aseveraciones que se puedan realizar en estas obras, ya que se refieren al grado de compromiso del hablante con las proposiciones del texto, esto es, al grado de importancia y veracidad que otorgan a sus afirmaciones, transmitiendo al/la lector/a que algunas cosas son más veraces y fiables que otras:

Las personas advierten, aunque no necesariamente de manera consciente, que algunas cosas que conocen son más certeras y que pueden apostar que son más verdaderas que otras, es decir, que no todo conocimiento es igualmente confiable. Así, un modo en que se puede calificar el conocimiento es por medio de una expresión que indica la evaluación que el hablante hace acerca de su grado de confiabilidad (Chafe 1986: 264).

$\mathrm{Al}$ mismo tiempo, deberemos subrayar aquellos enunciados que nos remitan a la idea de aprendizaje, de incorporar valores y capacidades. Para identificar este último aspecto, prestaremos especial atención a los infinitives fact, in fact, science, percent, surveys, study, culture, cultural, education, learn to, learn from, need to, listen to, ask, take, to be, real, reality... Una vez tengamos identificadas todas las cuestiones señaladas anteriormente, deberemos reflexionar sobre el modelo de mujer líder que se deriva de esos datos. Para esto, se identificarán en primer lugar los valores que se desprenden de esos mandatos. Más tarde, discerniremos si dichos valores son asociados a las mujeres de forma esencialista, o si son ofrecidos como valores que cualquier persona puede incorporar.

Por último, conseguiremos discernir qué tipo de discurso vertebra el modelo de mujer líder que se construye en este tipo de literatura, si se trata de un discurso transfomador, o de un discurso que esencializa y fundamenta en diferencias biológicas las diferencias entre mujeres y hombres. A su vez, iremos conformando el tipo de modelo de mujer líder que caracteriza a estas obras, y seremos capaces de responder qué quiere decir ser mujer líder en la empresa a través de este tipo de literatura. A continuación, se dispone un esquema de nuestra propuesta de aplicación de la Appraisal Theory al análisis de la literatura gerencial en base a los objetivos definidos en esta investigación: 


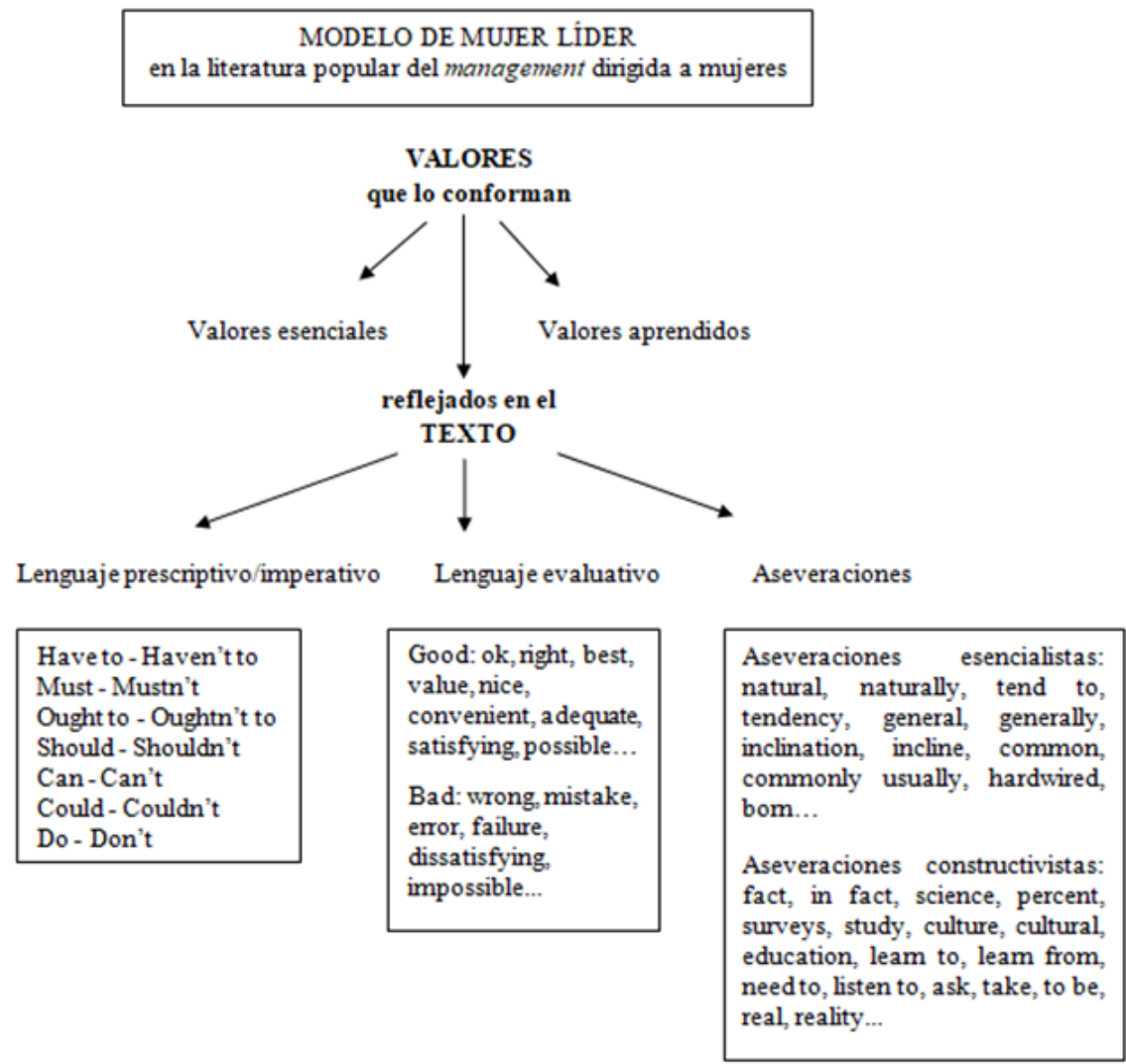

\section{SELECCIÓN DEL CORPUS}

Si partimos de la definición del corpus como "una colección finita de materiales, determinada previamente por el analista, con cierta (inevitable) arbitrariedad, sobre la cual se va a trabajar" (Barthes 1997: 80), éste debe reproducir el complejo sistema de oposiciones y confluencias que se daría en el total de dicha literatura. Además, tiene que presentar una homogeneidad según los criterios definidos en la investigación (Alonso y Fernández Rodríguez 2006b), que se explicitan a continuación.

En primer lugar, encontramos el criterio cronológico. Las obras estudiadas se editaron dentro del período 2010-2015, con el objeto de obtener un análisis de los valores más recientes en la construcción del modelo de mujer líder en la empresa. En segundo lugar, encontramos el criterio geográfico. La mayor parte 
de este tipo de obras son normalmente editadas en Norteamérica o el Reino Unido (Fernández Rodríguez 2007a). En tercer lugar, resulta importante atenerse a un criterio referido a los/as autores/as, su popularidad y ámbito profesional de procedencia. La relevancia de los/as autores/as resulta vital, ya que en un mundo como el del management, su reputación y fama es clave para la difusión de las ideas expuestas en este tipo de obras. Consecuentemente, consideramos que la popularidad es un rasgo esencial a tener en cuenta, ya que su carácter de gurús o expertos/as en temas empresariales otorga legitimidad a su discurso en el mundo de los negocios. En cuarto lugar, se presta especial atención a las críticas que hayan recibido las obras por parte de periódicos de gran tirada y autores/as de prestigio. En el caso de que no se pueda acceder al número de ventas de las obras en el momento de realizar la selección, nos centramos en el número de búsquedas realizadas a través del buscador de compras Amazon, plataforma que recoge los ejemplares de esta literatura para su venta y los clasifica en función de su popularidad/número de ventas entre los clientes.

Así pues, las obras que conforman la muestra a analizar se han seleccionado del corpus teórico inicial (150 obras $\left.{ }^{7}\right)$ en base a los criterios definidos previamente. Los cinco textos que conforman la muestra final han sido best sellers de la gestión empresarial o en su defecto, cuentan con gran presencia en los buscadores de ventas de libros online y buenas recomendaciones. A continuación, se dispone la distribución formal de dichas obras:

1. Mistakes I Made at Work: 5 Influential Women Reflect on What They Got Out of Getting It Wrong, de Jessica Bacal (2014).

2. Work with Me. How Gender Intelligence Can Help you Succeed at Work and in Life, de Barbara Annis y John Gray (2013).

3. How Women Lead: The 8 Essential Strategies Successful Women Know, de Sharon Hadary y Laura Henderson (2013).

4. Who Says It's a Man's World: The Girls' Guide to Corporate Domination, de Emily Bennington (2013).

5. Lean In: Women, Work and the Will to Lead, de Sheryl Sandberg (2013).

$\mathrm{Su}$ análisis nos permitirá extraer conclusiones sobre los valores que se encuentran presentes en dicha literatura en referencia a la presencia de mujeres en la empresa y su papel como líderes en la misma.

${ }^{7}$ Para seleccionar el corpus teórico del análisis se ha utilizado el buscador Amazon.com y sus listas de Best Sellers, tanto en una acepción genérica como en la categoría de Women \& Business definida por la web. Para acceder al listado del corpus teórico completo consultar Medina-Vicent (2018a). 


\section{RESULTADOS}

Durante el análisis se han encontrado gran cantidad de indicadores de lenguaje prescriptivo/imperativo, de aseveraciones de corte esencialista y de corte constructivista, pero no tantos del evaluativo. En el primer caso, el del lenguaje prescriptivo/imperativo, cabe añadir que no se ha encontrado ningún caso de ought to ni en su forma afirmativa ni en su forma negativa. Una de las explicaciones más coherentes para este fenómeno puede referirse a que estos textos hacen uso de un lenguaje informal para llegar con mayor rapidez a su público. Por esta razón, normalmente se utilizan fórmulas más coloquiales, hecho que explica la predominancia de las formas más informales que aquí tratamos.

A su vez, se ha observado que el lenguaje prescriptivo/imperativo se refiere sobre todo a cuestiones aspiracionales, lo que se debe y lo que no se debe hacer en vistas a alcanzar un objetivo. De forma continua se utilizan verbos que instan al/la lector/a a iniciar un cambio en su comportamiento o personalidad: have to be, have to be able, have to figure out, have to feel, have to get, have to realize, have to do, have to try.... Estos rasgos nos remiten a las semejanzas de la literatura gerencial con la literatura de autoayuda ${ }^{8}$, caracterizada por una psicologización de los problemas sociales que pasan a ser gestionados por las personas individuales (Papalini 2006, 2013, 2016).

En este sentido, y aunque la formula must se usa con menor frecuencia, cuando se usa, se suele acompañar del verbo to be. Esta realidad concuerda con el carácter aspiracional de la literatura popular del management, que pretende producir una transformación en el sujeto lector, que pasa de una situación a otra mediante un cambio de actitud o accionar derivado de la lectura de dichas obras. En este tipo de libros es solamente a través del cambio interno del/la lector/a donde se encuentra el potencial transformador, y esto se ejercita a través de su aspiración a cambiar de un estado a otro (Taylor, Pham, Rivkin, y Armor 1998). A continuación se disponen algunos ejemplos de lo comentado:

Be an initiator of change, not a follower (Hadary y Henderson 2013: 65). Be mindful of your stress triggers (Bennington 2013: 39).

Be your best (Bennington 2013: 213).

${ }^{8}$ El desarrollo de la literatura de autoayuda se produce con la emergencia de lo que la socióloga Eva Illouz denomina “capitalismo emocional". En sus obras Intimidades congeladas (2007) y La salvación del alma moderna (2010), trata el papel de las emociones en la construcción del capitalismo. Su tesis central se refiere a la necesidad que tiene el capitalismo de instaurar una cultura emocional en la vida de los individuos que reproduzca las lógicas de intercambio del sistema económico. Desde nuestro punto de vista, la literatura de autoayuda es un instrumento clave en la conformación de dicha cultura emocional pro-capitalista y en su divulgación entre la gente de a pie. La literatura managerial sería el eco que tiene esta lógica en la incorporación de la instrumentalización de las emociones dentro de la empresa 
But I also know that in order to continue to grow and challenge myself, $\underline{I}$ have to believe in my own abilities (Sandberg 2013: 38).

Based on your values, you have to decide what aspects of your life take precedence at different times (Hadary y Henderson 2013: 146).

You have to make one thing a priority and achieve balance that way, rather than trying to everything all at once (Bennington 2013: 31).

From an early age, girls get the message that they will have to choose between succeeding at work and being a good mother (Sandberg 2013: 92).

At the same time, we must be careful not to inject gender into every discussion (Sandberg 2013: 150).

Por otro lado, la fórmula should, tanto en su forma positiva como negativa, es muy utilizada, y en muchas ocasiones sirve para dar instrucciones sobre lecturas que se deberían realizar, o decisiones que se deberían tomar para iniciar dicho cambio. Lo mismo ocurre con las fórmulas can/can't y could/couldn't. Otro dato interesante lo encontramos en que se suelen utilizar con gran frecuencia las fórmulas negativas de todos los indicadores tratados hasta el momento, como por ejemplo la forma negativa don't. Desde nuestro punto de vista, esto resulta muy revelador, ya que en muchas ocasiones cuando se nos dice que no podemos hacer algo, los límites que se fijan con la prohibición delimitan con más fuerza aquello que somos y aquello que debemos llegar a ser, es decir, los límites de nuestra identidad. Teniendo en cuenta que las formas negativas son vitales cuando se nos dice lo que deberíamos hacer, identificarlas ha resultado muy revelador para nuestro estudio.

Whenever possible, women should substitute "we" for "I" (Sandberg 2013: 47).

But if you don't want to blur the line, for example, don't wear the low-cut shirts and high skirts (Bennington 2013: 67).

Don't be a victim when you don't have to be (Bennington 2013: 74).

First and foremost, don't! From the beginning of your career, do not be drawn in by the attraction of an affair, whether with a senior executive, a manager, a colleague, or someone who works for you (Hadary y Henderson 2013: 99).

Don't let the need to be seen as perfect by those you admire keep you from developing mentoring relationships (Bacal 2014: 245).

Understanding those lessons affirmed my belief that mistakes are nearly always learning opportunities and that you should only call them «failures» if you don't learn (Bacal 2014: 111).

Don't stop being a woman just because you are in a job (Hadary y Henderson 2013: 14).

Por otro lado, las formas referidas al lenguaje evaluativo no son tan predominantes como las anteriores. Además, cabe señalar la confusión que se desprende de su uso, es decir, habíamos determinado los indicadores para encontrar aquello que se evaluaba como malo o bueno en el comportamiento de las mujeres en la empresa: good, ok, right, best, value, nice, convenient, adequate, satisfying, possible, bad, wrong, mistake, error, failure, dissatisfying, impossible... No obstante, a la hora de aplicar la metodología al análisis de las obras, hemos visto que en 
muchas ocasiones se entremezclaban dichos indicadores, con lo que, aunque en una frase se pudiera encontrar la palabra good, podía ocurrir que el significado final de esa misma frase no estuviera relacionado con una acción positiva, sino al contrario. Se trata de una realidad que hemos tenido en cuenta a lo largo de todo el análisis y donde ha tenido que intervenir la capacidad interpretativa de nuestro papel como analista del discurso.

Además, esta idea nos remite a un rasgo de las obras que es la ambigüedad estratégica (Alvesson y Svenginsson 2003; Eisenberg 2009) que las caracteriza tanto en los términos que se manejan como en las posiciones sociales a las que remiten. La contradicción entre los consejos que se lanzan desde estas obras es una muestra clara de la complejidad que caracteriza a la presencia de las mujeres en el ámbito público y del trabajo. Dicha complejidad es fruto de la pervivencia de viejas dicotomías de género en un mundo en el que el mercado actúa como un agente disciplinador para los actores de la empresa, que se doblegan al principio de la accountability (Laval y Dardot 2013). De este modo, se hace hueco entre la sociedad civil el discurso del riesgo inherente a la existencia individual, por la que, si cada uno es responsable de su propio destino, la sociedad no le debe nada. De este modo, convertidos en agentes racionales, los sujetos deben moverse en el ámbito de la organización laboral reconvertido en un régimen de autodisciplina que manipula las instancias psíquicas del deseo y de la culpabilización.

Por último, encontramos las aseveraciones de corte esencialista o constructivista, con las que nos ocurre algo parecido a lo señalado en el párrafo anterior. Para identificar este tipo de fórmulas del lenguaje habíamos establecido diferentes indicadores como natural, naturally, tend to, fact, in fact, science, percent,... Sin embargo, el contenido que se desprende de los fragmentos identificados es difícil de clasificar como esencialismo o constructivismo, ya que se mueven en una ambigüedad que hace complicado poder establecer una conclusión estable de su carácter. Puede ocurrir que la autora esté recurriendo a un estudio científico o a cifras derivadas de la realización de encuestas - algo que se podría considerar como constructivista-, pero que la lectura que se desprenda del mismo sea de corte esencialista, tal y como se puede observar a continuación:

The IPL tends to be larger on the left - or logical, analytical, and objective - side of the brain in men, continuously prompting them to taking action, and with a fixed focus on task and achievement. Men tend to gauge their ability and measure their worth by their accomplishments and results. They feel great comfort and competence when solving problems in isolation and thinking things trough to their logical constructions (Annis y Gray 2013: 219).

When you look a little deeper, you start to recognize that the male model of work is primarily a product of the physiology of the male brain. The way men process work through individual decision making, singular focus, and immediate action parallels the way men instinctively process their world around them. And it's hard to change what's hardwired (Annis y Gray 2013: 46).

Without a doubt, social conditioning and natural temperament play a part 
in defining who we are and how we think and behave as individuals. Nevertheless, biological gender differences have been shown to be true in scientific $\underline{\text { studies }}$ based on large, diverse populations of men and women (Annis y Gray 2013: 64).

Se trata de esa ambigüedad que nos desconcierta como lectores/as y que dificulta el arrojar conclusiones definitivas sobre la literatura popular del management dirigida a mujeres en la cuestión de la predominancia del esencialismo o el constructivismo en torno a la cuestión de género. Sin embargo, es ese mismo carácter ambiguo el que nos hace decantarnos hacia la tendencia esencialista, ya que, por ejemplo, el uso que se hace en estas obras de los argumentos neurocientíficos siempre se dirigen a sustentar una ventaja competitiva de las mujeres en el desarrollo del liderazgo de corte biológico o esencialista (Medina-Vicent y Pallarés-Domínguez 2017). Por tanto, los valores expuestos en este tipo de obras en su asociación al modelo de liderazgo femenino estarían más cercanos a un discurso de carácter predominantemente esencialista.

\section{CONCLUSIONES}

La aplicación de la metodología de la Appraisal Theory al análisis de la evaluación y las prescripciones morales sobre el comportamiento de las mujeres líderes en la literatura managerial ha mostrado ser una herramienta flexible con ciertas limitaciones. En el estudio de los valores morales y las prescripciones que dictan cómo debería comportarse una mujer en el ámbito de la dirección empresarial, dicha metodología nos brinda el espacio de trabajo adecuado para identificar la "corrección" del comportamiento femenino en base a unas expectativas de comportamiento dicotómicas que perviven en el mundo de la gestión. Sin embargo, en la identificación de los indicadores encontramos ciertas limitaciones referidas sobre todo a las normas morales vigentes en el contexto en el que los textos adquieren sentido. Es en este aspecto en el que el/la analista, a la hora de aplicar la teoría de la valoración, necesita ir más allá de los indicadores y situar el sentido de su uso en un marco de sentido mayor referido a los discursos y las ideologías inscritas en los textos.

En conclusión, hemos podido comprobar que una idea central atraviesa todas las lecturas convirtiéndose en la columna vertebral del repertorio argumental de la literatura popular del management dirigida a mujeres que hemos dado en analizar. Nos estamos refiriendo a la ambiguiedad estratégica de los argumentos inscritos en estas obras, cuyo discurso por la igualdad resulta poco profundo y ampliamente despolitizado, y cuya visión de los roles de género resulta contradictoria con los principios de lucha social del feminismo (Reverter-Bañón 2001, 2016). Dicho carácter, como hemos comentado, tiene mucho que ver con los lazos que atan a esta literatura con la autoayuda (Siurana 2018), que conduce a una privatización de los problemas sociales, que se construyen como cuestiones de importancia psicoterapéutica, médica, espiritual o religiosa que ya no pueden ser 
enmarcadas como problemas de acción colectiva (Nehring, Alvarado, Hendriks, y Kerrigan 2016: 147).

Así pues, finalmente concluimos que sí se puede identificar una tendencia general a defender la existencia de un liderazgo femenino asociado al estereotipo de género femenino tradicional, que contiene un carácter esencialista y excluyente que reproduce las dicotomías de género y el modelo racional del homo oeconomicus, impidiendo el desarrollo de una gestión empresarial ética (Cortina 1994; Conill 2003; García-Marzá 2004). Un modelo de liderazgo que responda a la lógica neoliberal basada en los valores de la autorrealización, la autoexigencia y el emprendimiento no puede abrir paso a una sociedad más igualitaria, ni tampoco a un ámbito empresarial más ético, ya que anula completamente una visión colectiva de los problemas sociales, desarticulando cualquier posibilidad de lucha feminista. Las implicaciones éticas que tiene esta realidad en la consecución de la igualdad de género dentro de las organizaciones son claves, ya que si se promueven modelos de liderazgo de corte instrumental, se están fortaleciendo las estructuras de desigualdad de género que caracterizan al ámbito empresarial. Cualquier pretensión de transformación social, ya sea en la empresa o en cualquier otro espacio, lleva aparejado el análisis crítico de las relaciones de poder que reproducen dicha desigualdad.

En este sentido, el discurso analizado en el presente trabajo nos muestra que en lo que se refiere a la promoción de una verdadera igualdad de género en la empresa aún queda mucho por hacer, y lo que resulta más importante, la expansión de un discurso de corte esencialista sobre la presencia de las mujeres en la empresa nos revela que debemos mantenernos alerta sobre las derivas neoliberales que está tomando parte del feminismo en la actualidad (Medina-Vicent 2018b). Y es que, al formar parte de los discursos actuales del management (Fernández-Rodríguez y Medina-Vicent 2017), la concepción esencialista del liderazgo empresarial de las mujeres se está convirtiendo en una de las piedras angulares de la presencia de las mujeres en la empresa. Así pues, no es solamente preciso continuar analizando críticamente el ámbito de las organizaciones desde la perspectiva feminista, como ya se han encargado de hacer predecesoras como Marta Calas y Linda Smircich (1992a, 1992b), sino también rearticular el componente político del feminismo para así detectar las derivas neoliberales del mismo (Reverter-Bañón 2017).

\section{BIBLIOGRAFÍA}

ALONSO, L. E. (1998): La mirada cualitativa en sociología, Madrid, Fundamentos.

ALONSO, L. E., y FERNÁNDEZ RODRÍGUEZ, C. J. (2006): «Roland Barthes y el Análisis del Discurso», Empiria. Revista de Metodología de Ciencias Sociales, 12, pp. 11-35.

ALONSO, L. E., y FERNÁNDEZ RODRÍGUEZ, C. J. (2013): Los discursos del presente. Un análisis de los imaginarios sociales contemporáneos, Madrid, Siglo XXI. 
ALONSO, L. E., y FERNÁNDEZ RODRÍGUEZ, C. J. (2018): Poder y sacrificio. Los nuevos discursos de la empresa, Madrid, Siglo XXI.

ALVESSON, M., y SVENGINSSON, S. (2003): «Managers Doing Leadership: The Extra-Ordinarization of the Mundane», Human Relations, 56(12), pp. 1435-1459. https://doi.org/10.1177/00187267035612001

ALVESSON, M., y WILLMOTT, H. (1992): Critical Management Studies, London, Sage.

ANNIS, B., y GRAY, J. (2013): Work with Me: How Gender Intelligence Can Help You Succeed at Work and in Life, London, Piatkus.

BACAL, J. (2014): Mistakes I Made at Work, New York, Penguin Group.

BARTHES, R. (1997): La aventura semiológica, Barcelona, Paidós.

BENNINGTON, E. (2013): Who Says It's a Man's World. The Girl's Guide to Corporate Domination, New York, AMACOM.

BENSCHOP, Y., y DOOREWAARD, H. (1998): «Covered by Equality: The Gender Subtext of Organizations», Organization Studies, 19(5), pp. 787-805. https://doi. org/10.1177/017084069801900504

BOLTANSKI, L., y CHIAPELLO, È. (2002): El nuevo espíritu del capitalismo, Madrid, Akal.

CALAS, M. B., y SMIRCICH, L. (1992a): «Re-writing Gender into Organizational Theorizing: Directions from Feminist Perspectives», en Rethinking Organization: New Directions in Organization Theory and Analysis, London, Sage, pp. 227-253.

CALAS, M. B., y SMIRCICH, L. (1992b): «Using the "F" word: Feminist Theories and the Social Consequences of Organizational Research», en Gendering Organizational Theory and Analysis, Newbury Park, Sage.

CHAFE, W. (1986): «Evidentiality in English Conversation and Academic Writing», en Evidentiality: the Linguistic Coding of Epistemology, New Jersey, Praeger.

CHIAPELLO, Ė., y FAIRCLOUGH, N. (2002): «Understanding the New Management Ideology: A Transdisciplinary Contribution from Critical Discourse Analysis and New Sociology of Capitalism», Discourse \& Society, 13(2): pp. 185-208. doi:10.11 77/0957926502013002406.

CHRISTIE, F., y MARTIN, J. R. (1997): Genres and Institutions: Social Processes in the Workplace and School, London, Cassell.

CONILL, J. (2003): «Economía ética en la era de la información», en Construir confianza. Ética de la empresa en la sociedad de la información y las comunicaciones, Madrid, Trotta, pp. 75-96.

CORTINA, A. (1994): Ética de la empresa: claves para una nueva cultura empresarial, Madrid, Trotta.

DUE BILLING, Y. (2011): «Are Women in Management Victims of the Phantom of the Male Norm?», Gender, Work and Organization, 18(3), pp. 298-317. https://doi. org/10.1111/j.1468-0432.2010.00546.x

EGGINS, S., y SLADE, D. (1997): Analysing Casual Conversation, London, Cassell.

EISENBERG, E. M. (2009): «Ambiguity as Strategy in Organizational Communication», Communication Monographs, 51(3), pp. 227-242. https://doi. org/10.1080/03637758409390197

FAIRCLOUGH, N. (1992a): Discourse and Social Change, Cambridge, Polity Press.

FAIRCLOUGH, N. (1992b): «Discourse and Text: Linguistic and Intertextual Analysis within Discourse Analysis», Discourse \& Society, 3(2), pp. 193-217. https://doi. org/10.1177/0957926592003002004 
FAIRCLOUGH, N. (2003): Analysing Discourse: Textual Analysis for Social Research, New York, Routledge.

FERNÁNDEZ RODRÍGUEZ, C. J. (2007a): El discurso del Management: tiempo y narración, Madrid, Centro de Investigaciones Sociológicas.

FERNÁNDEZ RODRÍGUEZ, C. J. (2007b): «Postmodernidad y teoría crítica de la empresa. Una presentación de los Critical Management Studies», en Vigilar y organizar: una introducción a los Critical Management Studies, Madrid, Siglo XXI Editores, pp. 1-24.

Fernández Rodríguez, C. J. y MEDINA-VICENT, M. (2017): «Los nuevos discursos del management: difusión, impactos y resistencias», Recerca. Revista de pensament i anàlisi, 20, pp. 7-14. https://doi.org/10.6035/Recerca.2017.20.1

García-Marzá, D. (2004): Ética empresarial: del diálogo a la confianza, Madrid, Trotta.

HADARY, S., y HENDERSON, L. (2013): How Women Lead. The 8 Essential Strategies Successful Women Know, New York, McGraw-Hill.

HUCZYNSKI, A. (1993): Management Gurus: What Makes Them and how to Become One, London, Routledge.

IEDEMA, R., FEEZ, S., y WHITE, P. R. R. (1994): Media Literacy, Sidney, NSW Publications.

ILLOUZ, E. (2007): Intimidades congeladas, Buenos Aires, Katz Editores.

ILLOUZ, E. (2010): La salvación del alma moderna, Madrid, Katz Editores.

INSTITUTO NACIONAL DE ESTADÍSTICA (2017): Mujeres y hombres en España. Mujeres en altos cargos públicos y privados, Madrid, Gobierno de España.

KENNY, K., y FOTAKI, M. (2015): «From gendered organizations to compassionate borderspaces: Reading corporeal ethics with Bracha Ettinger», Organization, 22(2), pp. 183-199. https://doi.org/10.1177/1350508414558723

LAVAL, C., y DARDOT, P. (2013): La nueva razón del mundo. Ensayo sobre la sociedad neoliberal, Barcelona, Gedisa.

LISCHINSKY, A. (2008): «Examples as persuasive argument in popular management literature», Discourse \& Communication, 2(3), pp. 243-269. https://doi. org/10.1177/1750481308091907

MARTIN, J. R. (2000): «Beyond Exchange: Appraisal Systems in English», en Evaluation in Text. Authorial Stance and the Construction of Discourse, Oxford, Oxford University Press, pp. 142-175.

MARTIN, J. R., y ROSE, D. (2003): Working with Discourse: Meaning Beyond the Clause, London, Continuum.

MARTIN, J. R., y WHITE, P. R. R. (2005): The Language of Evaluation: The Appraisal in English, New York, Palgrave Macmillan.

MAVIN, S., GRANDY, G., y WILLIAMS, J. (2014): «Experiences of Women Elite Leaders Doing Gender: Intra-gender Micro-violence between Women», British Journal of Management, 25(3), pp. 439-455. https://doi.org/10.1111/1467-8551.12057

MEDINA-VICENT, M. (2018a): Género y management en el marco neoliberal. Un análisis crítico para la emergencia de liderazgos feministas, Castelló de la Plana, Universitat Jaume I.

MEDINA-VICENT, M. (2018b): «Flirting with Neoliberalism: The Transfiguration of Feminist Political Awareness», NORA - Nordic Journal of Feminist and Gender Research, 26(1), pp. 69-75. https://doi.org/10.1080/08038740.2018.1424728

MEDINA-VICENT, M. y PALLARÉS-DOMÍNGUEZ, D. (2017): «Las huellas del Neurosexismo en la literatura popular del management dirigida a mujeres», Política y sociedad, 54(3), pp. 683-705. 
MEDINA-VICENT, M. y REVERTER-BAÑÓN, S. (2016): «La perspectiva de género como una mirada crítica al mundo», Asparkía. Revista de Investigació Feminista, 29, pp. 11-16. https://doi.org/http://dx.doi.org/10.6035/Asparkia.2016.29.1

MORRISH, L., y SAUNTSON, H. (2013): «'Business-facing Motors for Economic Development': an Appraisal Analysis of Visions and Values in the Marketised UK University», Critical Discourse Studies, 10(1), pp. 61-80. https://doi.org/10.1080/1740 5904.2012.736698

NEHRING, D., ALVARADO, E., HENDRIKS, E. C., y KERRIGAN, D. (2016): Transnational Popular Psychology and the Global Self-Help Industry: The Politics of Contemporary Social Change, London, Palgrave Macmillan.

PAINTER, C. (2003): «Developing Attitude: An Ontogenetic Perspetive on Appraisal», Text, 23(2), pp. 183-209.

PAPALINI, V. (2006): «La subjetividad disciplinada: de la contracultura a la autoayuda», en La comunicación como riesgo: cuerpo y subjetividad, La Plata, Ediciones Al Margen, pp. 21-44.

PAPALINI, V. (2013): «Recetas para sobrevivir a las exigencias del neocapitalismo», Nueva Sociedad, 245, pp. 163-177.

PAPALINI, V. (2016): Garantías de felicidad: estudio sobre los libros de autoayuda. Buenos Aires, Adriana Hidalgo Editora.

REVERTER-BAÑÓN, S. (2001): «Feminismo y democracia: una crítica antifundamentalista», Recerca. Revista de pensament i anàlisis, 1, pp. 95-108.

REVERTER-BAÑÓN, S. (2016): «¿Es necesario el feminismo hoy?», en Investigació i gènere a la Universitat Jaume I, Castelló de la Plana, Publicacions de la Universitat Jaume I, pp. 14-35.

REVERTER-BAÑ́N, S. (2017): «Cosmopolitismo Feminista contra Globalización», Araucaria. Revista Iberoamericana de Filosofía, Política y Humanidades, 19, pp. 301-325. https://doi.org/10.12795/araucaria.2017.i37.15

Sandberg, S. (2013): Lean In. Women, Work and the Will to Lead, New York, Alfred A. Knopf.

SCHARFF, C. (2016): «The Psychic Life of Neoliberalism: Mapping the Contours of Entrepreneurial Subjectivity», Theory, Culture \& Society, 33(6), pp. 107-122. https://doi.org/10.1177/0263276415590164

SIURANA, J. C., (2018): Felicidad a golpe de autoayuda, Barcelona, Plaza y Valdés.

TAYLOR, S. E., PHAM, L. B., RIVKIN, I. D., y ARMOR, D. A. (1998): «Harnessing the Imagination: Mental Simulation, Self-Regulation, and Coping», American Psychologist, 53(4), pp. 429-439. https://doi.org/10.1037/0003-066X.53.4.429

VÁZQUEZ MAZZINI, M. (2017): «Entre la racionalidad instrumental y el «imaginario managerial». Estrategias didácticas en la enseñanza del Management», Recerca. Revista de pensament i anàlisi, 20, pp. 35-57. https://doi.org/10.6035/Recerca.2017.20.3

WHITE, P. R. R. (2000): "Dialogue and Inter-Subjectivity: Reinterpreting the Semantics of Modality and Hedging", en Working with Dialogue, Tubingen, Neimeyer, pp. 67-80.

WHITE, P. R. R. (2002): “Appraisal-the Language of Evaluation and Stance”, en Handbook of Pragmatics, Amsterdam, John Benjamins, pp. 1-23. 